\title{
Existence of Cascade Discrete-Continuous State Estimators for Systems on a Partial Order
}

\author{
Domitilla Del Vecchio and Richard M. Murray \\ Control and Dynamical Systems \\ California Institute of Technology \\ 1200 E California Boulevard, Mail Stop 107-81 \\ Pasadena, CA 91125 \\ \{ddomitilla, murray\}@cds.caltech.edu
}

\begin{abstract}
In this paper, a cascade discrete-continuous state estimator on a partial order is proposed and its existence investigated. The continuous state estimation error is bounded by a monotonically nonincreasing function of the discrete state estimation error, with both the estimation errors converging to zero. This work shows that the lattice approach to estimation is general as the proposed estimator can be constructed for any observable and discrete state observable system. The main advantage of using the lattice approach for estimation becomes clear when the system has monotone properties that can be exploited in the estimator design. In such a case, the computational complexity of the estimator can be drastically reduced and tractability can be achieved. Some examples are proposed to illustrate these ideas.
\end{abstract}

\section{Introduction}

The analysis of systems that show "hybrid" behavior is precious to several engineering areas. Embedded systems and complex systems such as the Internet, biological systems, multi-agent systems, and many others provide examples of such a hybrid behavior. The problem of estimating the state becomes relevant when asking to control these systems or to verify the correctness of their behavior as is in the case of air-traffic control systems ([11], [2]).

The coupling of continuous and discrete dynamics renders the analysis of these systems hard. As pointed out by Bemporad [3], one of the biggest issues is complexity. One of the sources of such complexity is the absence of mathematical tools able to handle a uniform analysis of both the logic evolution and of the continuous evolution. In Del Vecchio et al. [5], it was shown that a partial order on the discrete variables can be used in order to reduce complexity of the discrete state estimator and achieve scalability in the number of variables to be estimated. In this paper, similar ideas are applied in order to estimate the continuous and the discrete variables in a unified framework.

There is a wealth of research on hybrid observer design. The pioneering work of Caines [4] proposes the observer tree method for the estimation of the discrete state of a finite state machine. The observer tree method is used also in Balluchi et al. [1] for the estimation of the discrete state. The estimator proposed in this paper is similar to the 
decoupled estimator design proposed by [1], except that in the present work the continuous and the discrete states are estimated simultaneously and asymptotic convergence and thus tracking of the state is achieved. If the dimension of the discrete variables set is very large, the estimation problem relying on observer-tree methods becomes intractable. If the system has some order preserving properties with respect to a suitable partial order, the method that is proposed in this paper generates a computationally efficient estimator. As opposed to [12], which proposes to detect the discrete state change a posteriori, here estimation and tracking of the state is sought.

The basic assumption this paper relies on is that the discrete state can be estimated without the aid of the continuous state estimate. This way, the continuous state estimate can be driven by the discrete state estimate. Thus, a cascade discrete-continuous state estimator is constructed that achieves convergence of the estimation error to zero and thus tracks the value of the state. The estimator is constructed on a larger variable space equipped with a partial order, where the extended system has some properties that are referred to as order compatibility for the discrete state dynamics and induced order compatibility for the continuous state dynamics. The proposed estimator can be constructed for any system that is observable and discrete state observable, and thus the lattice approach to estimation is general. The main advantage of this method is clear when the system enjoys some monotonic properties that the estimator can exploit directly. In such a case the complexity of the estimator is drastically reduced and a scalability property can be achieved in the number of variables to be estimated. This is shown in simulation examples.

This paper is organized as follows. In Section 2, basic notions on partial orders and on observability are reviewed. In Section 3, the model is introduced. In Section 4, the estimation problem is stated formally, and a solution is proposed in Section 5. The existence result of the proposed estimator is in Section 6. To show the generality of the proposed estimation scheme, the estimator is constructed for three different examples in Section 7. Section 8 gives some computational complexity estimates that clearly show the cases where the developed approach reduces the computational burden.

\section{Basic concepts}

This section reviews basic notions on partial order theory and on observability of deterministic transition systems.

\subsection{Partial Orders}

A partial order is a set $\chi$ with a partial order relation " $\leq$ ", and it is denoted $(\chi, \leq)$. The join " $\vee$ " and the meet" $\wedge$ " of two elements $x$ and $w$ in $\chi$ are defined as $x \vee w=\sup \{x, w\}$ and $x \wedge w=\inf \{x, w\}$, if $S \subseteq \chi, \vee S=\sup S$ and $\wedge S=\inf S$, where $\sup \{x, w\}$ denotes the smallest element in $\chi$ that is bigger than both $x$ and $w$, and inf $\{x, w\}$ denotes the largest element in $\chi$ that is smaller than both $x$ and $w$. If $x<w$ and there is no other element in between $x$ and $w$, we write $x \ll w$.

Let $(\chi, \leq)$ be a partial order. If $x \wedge w \in \chi$ and $x \vee w \in \chi$ for any $x, w \in \chi$, then $(\chi, \leq)$ is a lattice. Let $(\chi, \leq)$ be a lattice and let $S \subseteq \chi$ be a non-empty subset of $\chi$. Then $(S, \leq)$ 
is a sublattice of $\chi$ if $a, b \in S$ implies that $a \vee b \in S$ and $a \wedge b \in S$. If any sublattice of $\chi$ contains its least and greatest elements, then $(\chi, \leq)$ is called complete. Given a complete lattice $(\chi, \leq)$, we will be concerned with a special kind of a sublattice called an interval sublattice defined as follows. Any interval sublattice of $(\chi, \leq)$ is given by $[L, U]=\{w \in \chi: L \leq w \leq U\}$ for $L, U \in \chi$. That is, this special sublattice can be represented by only two elements. For example, the intervals of $(\mathbb{R}, \leq)$ are just the familiar closed intervals on the real line. The cardinality of an interval sublattice $[L, U]$ is denoted $|[L, U]|$.

The power lattice of a set $\mathcal{U}$, denoted $(\mathcal{P}(\mathcal{U}), \subseteq)$, is given by the power set of $\mathcal{U}$, $\mathcal{P}(\mathcal{U})$ (the set of all subsets of $\mathcal{U}$ ), ordered according to the set inclusion $\subseteq$. The meet and join of the power lattice is given by intersection and union. The bottom element is the empty set, that is $\perp=\emptyset$, and the top element is $\mathcal{U}$ itself, that is $T=\mathcal{U}$.

Let $(P, \leq)$ and $(Q, \leq)$ be partially ordered sets. A map $f: P \rightarrow Q$ is (i) an order preserving map if $x \leq w \Longrightarrow f(x) \leq f(w)$; (ii) an order embedding if $x \leq w \Longleftrightarrow$ $f(x) \leq f(w)$; (iii) an order isomorphism if it is order embedding and it maps $P$ onto $Q$.

A partial order induces a notion of distance between elements in the space. In this paper, the distance function on a partial order is defined as follows.

Definition 1. (Distance on a partial order) Let $(P, \leq)$ be a partial order. A distance $d$ on $(P, \leq)$ is a function $d: P \times P \rightarrow \mathbb{R}$ such that the following properties are verified:

(i) $d(x, y) \geq 0$ for any $x, y \in P$ and $d(x, y)=0$ if and only if $x=y$;

(ii) $d(x, y)=d(y, x)$;

(iii) if $x \leq y \leq z$ then $d(x, y) \leq d(x, z)$.

Because this paper is concerned with a partial order on the space of the discrete variables as well as with a partial order on the space of the continuous variables, it is useful to introduce the Cartesian product of two partial orders (see [9] for example).

Definition 2. (Cartesian product of partial orders) Let $\left(P_{1}, \leq\right)$ and $\left(P_{2}, \leq\right)$ be two partial orders. Their Cartesian product is given by $\left(P_{1} \times P_{2}, \leq\right)$, where $P_{1} \times P_{2}=\{(x, y) \mid x \in$ $P_{1}$ and $\left.y \in P_{2}\right\}$ and $(x, y) \leq\left(x^{\prime}, y^{\prime}\right)$ if and only if $x \leq x^{\prime}$ and $y \leq y^{\prime}$. For any $\left(p_{1}, p_{2}\right) \in$ $P_{1} \times P_{2}$ the standard projections $\pi_{1}: P_{1} \times P_{2} \rightarrow P_{1}$ and $\pi_{2}: P_{1} \times P_{2} \rightarrow P_{2}$ are such that $\pi_{1}\left(p_{1}, p_{2}\right)=p_{1}$ and $\pi_{2}\left(p_{1}, p_{2}\right)=p_{2}$.

\subsection{Deterministic Transition Systems and Observability}

The class of systems this work is concerned with are deterministic, infinite state systems with output defined as follows. A deterministic transition system (DTS) is the tuple $\Sigma=(S, \mathcal{Y}, F, g)$, where $S$ is a set of states with $s \in S ; \mathcal{Y}$ is a set of outputs with $y \in \mathcal{Y} ; F: S \rightarrow S$ is the state transition function; $g: S \rightarrow \mathcal{Y}$ is the output function. An execution of $\Sigma$ is any sequence $\sigma=\{s(k)\}_{k \in \mathbb{N}}$ such that $s(0) \in S$ and $s(k+1)=F(s(k))$ for all $k \in \mathbb{N}$. The set of all executions of $\Sigma$ is denoted $\mathcal{E}(\Sigma)$.

Definition 3. (Observability) The deterministic transition system $\Sigma=(S, \mathcal{Y}, F, g)$ is said to be observable if any two different executions $\sigma_{1}, \sigma_{2} \in \mathcal{E}(\Sigma)$ are such that there exists a $k$ such that $g\left(\sigma_{1}(k)\right) \neq g\left(\sigma_{2}(k)\right)$.

In the following section, the class of observable deterministic transition systems is restricted to those that are also discrete state observable. 


\section{The Model}

In this section, the distinction between the portion of the state that takes values in a finite set and the portion that takes values in an infinite possibly dense set is explicitly made. For a system $\Sigma=(S, \mathcal{Y}, F, g)$, suppose that $S=\mathcal{U} \times \mathcal{Z}$ with $\mathcal{U}$ a finite set, and $\mathcal{Z}$ an infinite possibly dense set; $F=(f, h)$, where $f: \mathcal{U} \times \mathcal{Y} \rightarrow \mathcal{U}$ and $h: \mathcal{U} \times \mathcal{Z} \rightarrow \mathcal{Z}$; $g: \mathcal{U} \times \mathcal{Z} \rightarrow \mathcal{Y}$ is the output map. These systems have the form

$$
\begin{aligned}
\alpha(k+1) & =f(\alpha(k), y(k)) \\
z(k+1) & =h(\alpha(k), z(k)) \\
y(k) & =g(\alpha(k), z(k)),
\end{aligned}
$$

and they are referred to as the tuple $\Sigma=(\mathcal{U} \times \mathcal{Z}, \mathcal{Y},(f, h), g)$. The function $f$ that updates the discrete variable $\alpha$ can be represented by a set of logic statements, or by a look-up table or recursive formula as is the case of finite state machines ([8]). For each value of $\alpha$, the equation (2) is a difference equation. Before stating the problem in more detail, an additional definition is given.

Definition 4. (Discrete state observability) The system $\Sigma=(\mathcal{U} \times \mathcal{Z}, \mathcal{Y},(f, h), g)$ is said to be discrete state observable if for any execution with output sequence $\{y(k)\}_{k \in \mathbb{N}}$, the following are verified

(i) The set of $\alpha$ compatible with the pair $(y(k), y(k+1))$, that is $\{\alpha \in \mathcal{U} \mid y(k)=$ $g(\alpha, z(k))$ and $y(k+1)=g(f(\alpha, y(k)), h(\alpha, z(k)))\}:=\mathcal{S}(k)$ does not depend on $z(k) ;$

(ii) if two executions $\sigma_{1}=\left\{\alpha_{1}(k), z_{1}(k)\right\}_{k \in \mathbb{N}}$ and $\sigma_{2}=\left\{\alpha_{2}(k), z_{2}(k)\right\}_{k \in \mathbb{N}}$ are such that the sequences $\left\{\alpha_{1}(k)\right\}_{k \in \mathbb{N}} \neq\left\{\alpha_{2}(k)\right\}_{k \in \mathbb{N}}$, then there is $k>0$ such that $\alpha_{1}(k) \in \mathcal{S}(k)$ and $\alpha_{2}(k) \notin \mathcal{S}(k)$.

Item (i) is trivially verified if $g(\alpha, z)=\left(g_{\alpha}(\alpha), g_{z}(\alpha, z)\right)$, where $g_{\alpha}: \mathcal{U} \rightarrow\left\{Y_{1}, Y_{2}, \ldots, Y_{m}\right\}$ partitions the set $\mathcal{U}$ in equivalence classes. We allow two steps in order to have an equivalence class that is independent of $z(k)$, as this is often the case when $\alpha$ acts in the $z$ dynamics. From this definition, it follows that a discrete state observable system admits a discrete state estimator that does not involve the continuous state estimate. This property will allow to construct a cascade discrete-continuous state estimator as defined in the following section.

\section{Problem Statement}

Consider the deterministic transition system $\Sigma=(\mathcal{U} \times \mathcal{Z}, \mathcal{Y},(f, h), g)$, with the output sequence $\{y(k)\}_{k \in \mathbb{N}}$. It is desirable to determine and track the value of the current state $(\alpha(k), z(k))$ of the system. This is more formally stated in the following problem.

Problem 1. (Cascade discrete-continuous state estimator) Given the deterministic transition system $\Sigma=(\mathcal{U} \times \mathcal{Z}, \mathcal{Y},(f, h), g)$, find functions $f_{1}, f_{2}, f_{3}, f_{4}, f_{5}$ with $f_{1}$ : $\chi \times \mathcal{Y} \times \mathcal{Y} \rightarrow \chi, f_{2}: \chi \times \mathcal{Y} \times \mathcal{Y} \rightarrow \chi, f_{3}: \mathcal{L} \times \chi \times \mathcal{Y} \times \mathcal{Y} \rightarrow \mathcal{L}, f_{4}: \mathcal{L} \times \chi \times \mathcal{Y} \times \mathcal{Y} \rightarrow \mathcal{L}$, 
$f_{5}: \mathcal{L} \rightarrow \mathcal{Z}_{E}$, with $\mathcal{U} \subseteq \chi,(\chi, \leq)$ a lattice, $\mathcal{Z} \subseteq \mathcal{Z}_{E}$ with $\left(\mathcal{Z}_{E}, \leq\right)$ a lattice, $\chi \times \mathcal{Z}_{E} \subseteq \mathcal{L}$, $(\mathcal{L}, \leq)$ a lattice, such that the update laws

$$
\begin{aligned}
L(k+1) & =f_{1}(L(k), y(k), y(k+1)) \\
U(k+1) & =f_{2}(U(k), y(k), y(k+1)) \\
q_{L}(k+1) & =f_{3}\left(q_{L}(k), L(k), y(k), y(k+1)\right) \\
q_{U}(k+1) & =f_{4}\left(q_{U}(k), U(k), y(k), y(k+1)\right)
\end{aligned}
$$

with $z_{L}(k)=f_{5}\left(q_{L}(k)\right)$ and $z_{U}(k)=f_{5}\left(q_{U}(k)\right)$, where $L(k), U(k) \in \chi, L(0):=\wedge \chi$, $U(0):=\bigvee \chi, q_{L}(k), q_{U}(k) \in \mathcal{L}, q_{L}(0)=\bigwedge \mathcal{L}, q_{U}(0)=\bigvee \mathcal{L}$, and $z_{L}(k), z_{U}(k) \in \mathcal{Z}_{E}$, have the following properties

(i) $L(k) \leq \alpha(k) \leq U(k)$ (correctness);

(ii) $|[L(k+1), U(k+1)]| \leq|[L(k), U(k)]|$ (non-increasing error);

(iii) There exists $k_{0}>0$ such that $[L(k), U(k)] \cap \mathcal{U}=\alpha(k)$ for any $k \geq k_{0}$ (convergence);

(i') $z_{L}(k) \leq z(k) \leq z_{U}(k)$;

(ii') there is a nonnegative function $V: \mathbb{N} \rightarrow \mathbb{R}$ such that $d\left(z_{L}(k), z_{U}(k)\right) \leq V(k)$, with $V(k+1) \leq V(k)$

(iii') There exists $k_{0}^{\prime}>k_{0}$ such that $d\left(z_{L^{\prime}}(k), z_{U^{\prime}}(k)\right)=0$ for any $k \geq k_{0}^{\prime}$, where $L^{\prime}=$ $\bigwedge([L, U] \cap \mathcal{U}), U^{\prime}=\bigvee([L, U] \cap \mathcal{U}), z_{L^{\prime}}(k)=f_{5}\left(q_{L^{\prime}}(k)\right), z_{U^{\prime}}(k)=f_{5}\left(q_{U^{\prime}}(k)\right), q_{L^{\prime}}(k+$ $1)=f_{3}\left(q_{L^{\prime}}(k), L^{\prime}(k), y(k), y(k+1)\right)$, and $q_{U^{\prime}}(k+1)=f_{4}\left(q_{U^{\prime}}(k), U^{\prime}(k), y(k), y(k+1)\right)$, with $q_{L^{\prime}}(0)=q_{L}(0)$ and $q_{U^{\prime}}(0)=q_{U}(0)$, for some distance function " $d$ ".

The update laws (3) are in cascade form as the variables $L$ and $U$ are updated on the basis of their previous values and on the basis of the output, while the variables $q_{L}$ and $q_{U}$ are updated on the basis of their previous values and on the basis of the values of $L$ and $U$ respectively. Note that the lower and the upper bound estimates of $z(k)$ are outputs of the laws that update $q_{L}(k)$ and $q_{U}(k)$, which lie in the space $\mathcal{L}$. Properties (iii) and (iii') roughly ask that the lower and upper bounds shrink to $\alpha(k)$ and $z(k)$. Property (ii') gives a monotonic bound on the continuous variable estimation error.

Note that the distance function " $d$ " has been left unspecified for the moment, as its form depends on the particular partial order chosen $\left(\mathcal{Z}_{E}, \leq\right)$. In the case in which $\mathcal{Z}=\mathcal{Z}_{E}$ and the order is established component-wise, the distance can be the classical euclidean distance. In the following section, a solution to the Problem 1 is proposed.

\section{Estimator Construction}

Given the deterministic transition system $\Sigma=(\mathcal{U} \times \mathcal{Z}, \mathcal{Y},(f, h), g)$, a set of sufficient conditions that allow a solution to Problem 1 is provided. With this respect, some definitions involving the extension of the system $\Sigma$ to a lattice are useful.

Definition 5. (System extension) Consider the system $\Sigma=(\mathcal{U} \times \mathcal{Z}, \mathcal{Y},(f, h), g)$. Let $(\chi, \leq),\left(\mathcal{Z}_{E}, \leq\right)$, and $(\mathcal{L}, \leq)$ be lattices with $\mathcal{U} \subseteq \chi, \mathcal{Z} \subseteq \mathcal{Z}_{E}$, and $\chi \times \mathcal{Z}_{E} \subseteq \mathcal{L}$. An extension of $\Sigma$ on the lattice $(\mathcal{L}, \leq)$ is given by $\tilde{\Sigma}=(\mathcal{L}, \mathcal{Y}, \tilde{F}, \tilde{g})$ such that

(i) $\tilde{F}: \mathcal{L} \times \mathcal{Y} \rightarrow \mathcal{L}$ and $\left.\tilde{F}\right|_{\mathcal{U} \times \mathcal{Z} \times \mathcal{Y}}=(f, h)$, and $\mathcal{L}-(\mathcal{U} \times \mathcal{Z})$ is invariant under $\tilde{F}$; 
(ii) $\left.\tilde{F}\right|_{\chi \times \mathcal{Z}_{E} \times y}=(\tilde{f}, \tilde{h})$ where $\tilde{f}: \chi \times \mathcal{Y} \rightarrow \chi,\left.\tilde{f}\right|_{\mathcal{U} \times y}=f, \tilde{h}: \chi \times \mathcal{Z}_{E} \rightarrow \mathcal{Z}_{E}$, and $\tilde{h} \mid \mathcal{U} \times \mathcal{Z}=h$

(iii) $\tilde{g}: \mathcal{L} \rightarrow \mathcal{Y}$ and $\left.\tilde{g}\right|_{\mathcal{U} \times \mathcal{Z}}=g$;

(iv) for any $q \in \mathcal{L}$ there exist $\left(w_{1}, \bar{z}_{1}\right),\left(w_{2}, \bar{z}_{2}\right) \in \chi \times \mathcal{Z}_{E}$ such that $\left(w_{1}, \bar{z}_{1}\right) \leq q \leq\left(w_{2}, \bar{z}_{2}\right)$, where $a_{L}(q):=\max _{(\mathcal{L}, \leq)}\left\{(w, \bar{z}) \in \chi \times \mathcal{Z}_{E} \mid q \geq(w, \bar{z})\right\}$ and $a_{U}(q):=\min _{(\mathcal{L}, \leq)}\{(w, \bar{z}) \in$ $\left.\chi \times \mathcal{Z}_{E} \mid q \leq(w, \bar{z})\right\}$

Item (iv) of the above definition establishes that the chosen lattices are such that any element in $\mathcal{L}$ that is not in $\chi \times \mathcal{Z}_{E}$ can be approximated by two elements in $\chi \times \mathcal{Z}_{E}$, $a_{L}(q)$ and $a_{U}(q)$. These are the lower and upper approximation of $q$ respectively. Note that if $q \in \chi \times \mathcal{Z}_{E}$, then $a_{L}(q)=a_{U}(q)=q$. The next definition links the discrete state dynamics of $\tilde{\Sigma}$ with the partial order $(\chi, \leq)$.

Definition 6. (Order compatibility) The pair $(\tilde{\Sigma},(\chi, \leq))$ is said to be order compatible if the following are verified

(i) $\{w \in \chi \mid y(k+1)=\tilde{g}(\tilde{f}(w, y(k)), \tilde{h}(w, z(k)))$ and $y(k)=\tilde{g}(w, z(k))\}=\left[l_{w}(k), u_{w}(k)\right]$ for $l_{w}(k), u_{w}(k) \in \chi$;

(ii) $\tilde{f}:\left(\left[l_{w}(k), u_{w}(k)\right], y(k)\right) \rightarrow\left[\tilde{f}\left(l_{w}(k), y(k)\right), \tilde{f}\left(u_{w}(k), y(k)\right)\right]$ is order isomorphic.

Item (i) in the above definition establishes that the set of $w \in \chi$ compatible with the pair $(y(k), y(k+1))$ for any execution $\sigma$ with output sequence $\{y(k)\}_{k \in \mathbb{N}}$ is a sublattice interval in $\chi$. Note that $\mathcal{S}(k)=\left[l_{w}(k), u_{w}(k)\right] \cap \mathcal{U}$ by definition. Two steps $k, k+1$ are allowed to obtain a set of $w \in \chi$ compatible with the output that does not depend on the values of $z$. For the construction of a cascade discrete-continuous state estimator, the case in which the partial order $(\mathcal{L}, \leq)$ is induced by the partial order $(\chi, \leq)$ by means of the system dynamics is of interest. Thus, a new notion of order compatibility is introduced in the next definition.

Definition 7. (Induced order compatibility) The pair $(\tilde{\Sigma},(\mathcal{L}, \leq))$ is said to be induced order compatible if

(i) for any $w_{1} \leq w_{2}$ in $\left[l_{w}(k), u_{w}(k)\right]$, there are $l_{q}\left(k, w_{1}\right), u_{q}\left(k, w_{2}\right) \in \mathcal{L}$ such that

$$
\begin{array}{r}
\left\{q \in \mathcal{L} \mid \pi_{1} \circ a_{L}(q)=w_{1}, \pi_{1} \circ a_{U}(q)=w_{2}, y(k+1)=\tilde{g}(\tilde{F}(q, y(k))), \text { and } y(k)=\tilde{g}(q)\right\} \\
\subseteq\left[l_{q}\left(k, w_{1}\right), u_{q}\left(k, w_{2}\right)\right] ;
\end{array}
$$

(ii) $a_{L}\left(l_{q}\left(k, w_{1}\right)\right)=\left(w_{1}, l_{z}\left(k, w_{1}\right)\right)$ and $a_{U}\left(u_{q}\left(k, w_{2}\right)\right)=\left(w_{2}, u_{z}\left(k, w_{2}\right)\right)$, for $l_{z}\left(k, w_{1}\right), u_{z}\left(k, w_{2}\right)$ $\in \mathcal{Z}_{E}$;

(iii) $\tilde{F}:\left(\left[l_{q}\left(k, w_{1}\right), u_{q}\left(k, w_{2}\right)\right], y(k)\right) \rightarrow\left[\tilde{F}\left(l_{q}\left(k, w_{1}\right), y(k)\right), \tilde{F}\left(u_{q}\left(k, w_{2}\right), y(k)\right)\right]$ is order preserving, and $\tilde{F}:\left(\alpha \times\left[l_{z}(k, \alpha), u_{z}(k, \alpha)\right], y(k)\right) \rightarrow\left[\tilde{F}\left(\alpha, l_{z}(k, \alpha), y(k)\right)\right.$, $\left.\tilde{F}\left(\alpha, u_{z}(k, \alpha), y(k)\right)\right]$ is order isomorphic;

(iv) for any $[L, U] \subseteq\left[l_{w}(k), u_{w}(k)\right]$

$$
d\left(\pi_{2} \circ a_{L} \circ \tilde{F}\left(l_{q}(k, L), y(k)\right), \pi_{2} \circ a_{U} \circ \tilde{F}\left(u_{q}(k, U), y(k)\right)\right) \leq \gamma(|[L, U]|),
$$

for some distance function " $d$ ", $\gamma: \mathbb{N} \rightarrow \mathbb{R}$ a monotonic function of its argument. 
Item (i) of this definition means that a sublattice interval in $(\chi, \leq)$ compatible with the output pair $(y(k), y(k+1))$ induces a sublattice interval in $(\mathcal{L}, \leq)$ corresponding to the same output pair. Item (ii) specifies that such output interval is approximated by the Cartesian product of two sublattice intervals in $(\chi, \leq)$ and in $\left(\mathcal{Z}_{E}, \leq\right)$. Item (iii) establishes the usual order preserving properties of the extension, and item (iv) establishes that the size of the interval lattice in $\left(\mathcal{Z}_{E}, \leq\right)$ induced by an interval $[L, U] \in \chi$ increases with the size of $[L, U]$. A solution to the Problem 1 is proposed by the following theorem.

Theorem 1. Given the system $\Sigma=(\mathcal{U} \times \mathcal{Z}, \mathcal{Y},(f, h), g)$, assume that there are lattices $(\chi, \leq),\left(\mathcal{Z}_{E}, \leq\right)$, and $(\mathcal{L}, \leq)$, with $\mathcal{U} \subseteq \chi, \mathcal{Z} \subseteq \mathcal{Z}_{E}$, and $\chi \times \mathcal{Z}_{E} \subseteq \mathcal{L}$ such that the pairs $(\tilde{\Sigma},(\chi, \leq))$ and $(\tilde{\Sigma},(\mathcal{L}, \leq))$ are order compatible and induced order compatible respectively. Then a solution to Problem 1 is provided by

$$
\begin{aligned}
L(k+1) & =\tilde{f}\left(l_{w}(k) \vee L(k), y(k)\right) \\
U(k+1) & =\tilde{f}\left(u_{w}(k) \wedge U(k), y(k)\right) \\
q_{L}(k+1) & =\tilde{F}\left(q_{L}(k) \vee l_{q}\left(k, l_{w}(k) \vee L(k)\right), y(k)\right) \\
q_{U}(k+1) & =\tilde{F}\left(q_{U}(k) \wedge u_{q}\left(k, u_{w}(k) \wedge U(k)\right), y(k)\right) .
\end{aligned}
$$

with $z_{L}(k)=\pi_{2} \circ a_{L}\left(q_{L}(k)\right)$ and $z_{U}(k)=\pi_{2} \circ a_{U}\left(q_{U}(k)\right)$.

Proof. The idea of the proof is analogous to the one proposed in [7]. Here, a sketch is provided, which highlights the differences due to the more general framework considered in this paper. For the proof of (i)-(ii)-(iii), the reader is deferred to [5]. Define $U^{*}=u_{w}(k) \wedge U(k), L^{*}=l_{w}(k) \vee L(k), q_{U}^{*}=q_{U}(k) \wedge l_{q}\left(k, U^{*}\right)$, and $q_{L}^{*}=q_{L}(k) \vee l_{q}\left(k, L^{*}\right)$. The dependence of $u_{q}$ and $l_{q}$ on their arguments is omitted, as well as the dependence of $\tilde{F}$ on $y$.

Proof of (i'). By using induction argument on $k$ and exploiting the order preserving property of $\tilde{F}$, one can show that $q_{L}(k) \leq(\alpha(k), z(k)) \leq q_{U}(k)$ (see Figure 1) for any $k$. By the the fact that $\pi_{2} \circ a_{L}$ and $\pi_{2} \circ a_{U}$ are order preserving functions, (i') follows (see Figure 1).

Proof of (ii'). Using the order preserving property of $\tilde{F}$, of $\pi_{2} \circ a_{L}$, and of $\pi_{2} \circ$ $a_{U}$, one deduces that $z_{L}(k+1) \geq \pi_{2} \circ a_{L} \circ \tilde{F}\left(l_{q}\left(k, L^{*}\right)\right)$ and $z_{U}(k+1) \leq \pi_{2} \circ a_{U} \circ$ $\tilde{F}\left(u_{q}\left(k, U^{*}\right)\right)$ (see Figure 1). By exploiting the property (iii) of the distance function in Definition 1, and the property (iv) given in Definition 7, one can infer that $d\left(z_{L}(k+\right.$ $\left.1), z_{U}(k+1)\right) \leq \gamma\left(\left|\left[L^{*}, U^{*}\right]\right|\right)$. Since $\tilde{f}$ is order isomorphic, it follows that $\left|\left[L^{*}, U^{*}\right]\right|=$ |[ $\left.\tilde{f}\left(L^{*}, y\right), \tilde{f}\left(U^{*}, y\right)\right] \mid$. Thus, (ii') of Problem 1 is satisfied with $V(k)=\gamma(|[L(k), U(k)]|)$.

Proof of (iii'). For $k>k_{0}, L^{\prime}(k)=\alpha(k)=U^{\prime}(k)$ as $[L(k), U(k)] \cap \mathcal{U}=\alpha(k)$. As a consequence, $q_{L^{\prime}}(k+1)=\tilde{F}\left(q_{L^{\prime}}(k) \vee l_{q}(k, \alpha(k))\right)$ and $q_{U^{\prime}}(k+1)=\tilde{F}\left(q_{U^{\prime}}(k) \vee u_{q}(k, \alpha(k))\right)$, where $l_{q}(k, \alpha)=\left(\alpha, l_{z}(k, \alpha)\right)$ and $u_{q}(k, \alpha)=\left(\alpha, u_{z}(k, \alpha)\right)$. One then uses the facts that $\left(\alpha, l_{z}(k, \alpha)\right) \leq q_{L^{\prime}}(k) \vee l_{q}(k, \alpha(k)), q_{U^{\prime}}(k) \vee u_{q}(k, \alpha(k)) \leq\left(\alpha, u_{z}(k, \alpha)\right)$, the fact that $\tilde{F}$ : $\left(\alpha \times\left[l_{z}(k, \alpha), u_{z}(k, \alpha)\right]\right) \rightarrow\left[\tilde{F}\left(\alpha, l_{z}(k, \alpha)\right), \tilde{F}\left(\alpha, u_{z}(k, \alpha)\right)\right]$ is order isomorphic, and the fact that $\mathcal{L}-(\mathcal{U} \times \mathcal{Z})$ is invariant under $\tilde{F}$. Proceeding by contradiction, if for any $k$ there are $\left(\alpha^{\prime}, z_{1}^{\prime}\right),\left(\alpha^{\prime}, z_{2}^{\prime}\right)$ in $\left[q_{L^{\prime}}(k), q_{U^{\prime}}(k)\right] \cap(\mathcal{U} \times \mathcal{Z})$ that are compatible with the output, there must be $\left(\alpha, z_{1}\right),\left(\alpha, z_{2}\right) \in\left[q_{L^{\prime}}(k-1), q_{U^{\prime}}(k-1)\right] \cap(\mathcal{U} \times \mathcal{Z})$ such that $\left(\alpha^{\prime}, z_{1}^{\prime}\right)=F\left(\alpha, z_{1}\right)$ and $\left(\alpha^{\prime}, z_{2}^{\prime}\right)=F\left(\alpha, z_{2}\right)$. Also, $\left(\alpha, z_{1}\right),\left(\alpha, z_{2}\right)$ are compatible with the output as well (see 


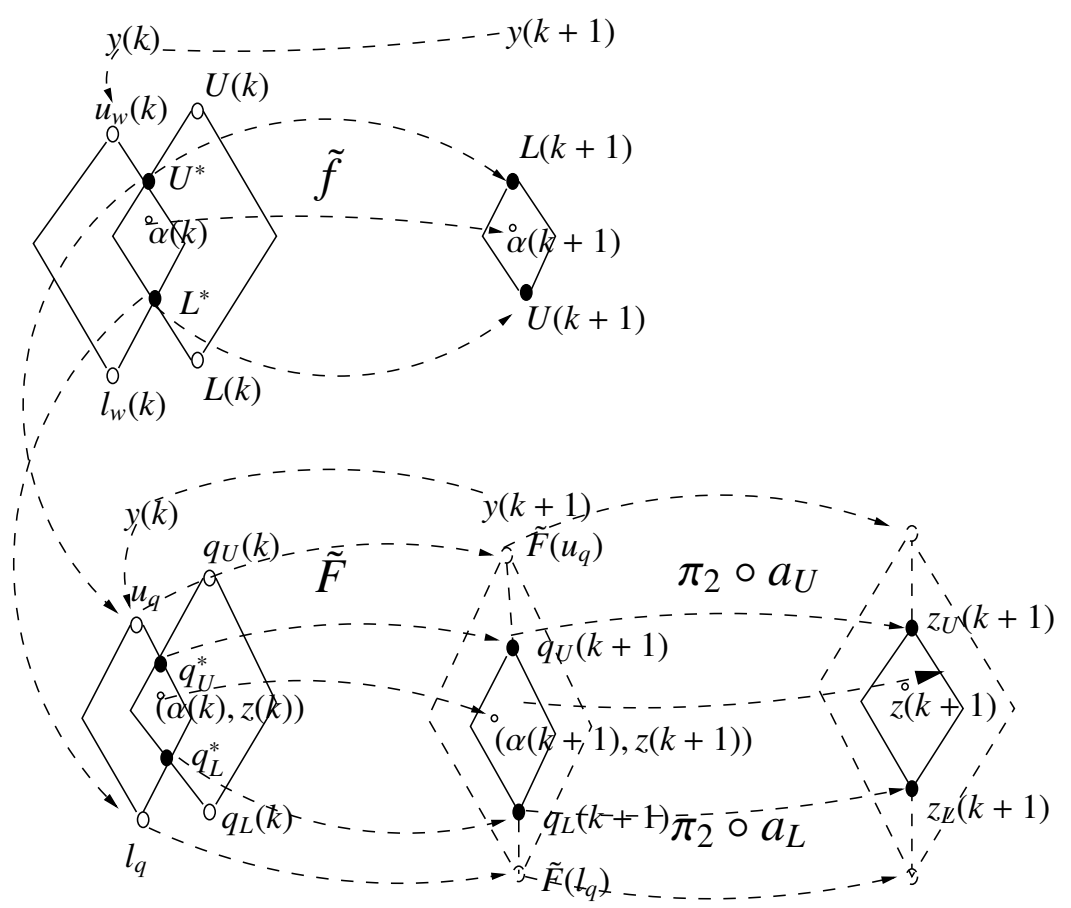

Fig. 1. Hasse diagrams representing the updates of the estimator in Theorem 1.

Figure 1). Since this is true for any $k$, one can construct two executions of $\Sigma$ that are different and share the same output sequence. This contradicts observability of $\Sigma$. Then there must be $k>k_{0}$ such that $\left[q_{L^{\prime}}(k), q_{U^{\prime}}(k)\right] \cap(\mathcal{U} \times \mathcal{Z})=(\alpha(k), z(k))$, and therefore $z_{L^{\prime}}(k)=z_{U^{\prime}}(k)=z(k)$.

In the following section, conditions in order to verify the assumptions needed for the construction of the estimator given in Theorem 1 are given. I particular, observability and discrete state observability are sufficient conditions for the estimator construction, and therefore the proposed estimation approach on a lattice is general.

\section{Estimator Existence}

The following theorem shows that if the system $\Sigma$ is observable and discrete state observable, the lattices $(\mathcal{L}, \leq),\left(\mathcal{Z}_{E}, \leq\right)$, and $(\chi, \leq)$ introduced in the previous section exist, such that the extended system is both order compatible with $(\chi, \leq)$ and induced order compatible with $(\mathcal{L}, \leq)$.

Theorem 2. Assume that the system $\Sigma=(\mathcal{U} \times \mathcal{Z}, \mathcal{Y},(f, h), g)$ is observable and discrete state observable. Then there exist lattices $(\chi, \leq),\left(\mathcal{Z}_{E}, \leq\right),(\mathcal{L}, \leq)$ with $\mathcal{U} \subseteq \chi, \mathcal{Z} \subseteq \mathcal{Z}_{E}$, and $\chi \times \mathcal{Z}_{E} \subseteq \mathcal{L}$, and an extension $\tilde{\Sigma}$ of $\Sigma$ on $(\mathcal{L}, \leq)$ that is order compatible with $(\chi, \leq)$ and induced order compatible with $(\mathcal{L}, \leq)$. 
Proof. To prove that discrete state observability implies the existence of a lattice $(\chi, \leq$ ) and an extension on $(\mathcal{L}, \leq)$ of $\Sigma$ that is order compatible with $(\chi, \leq)$, the reader is deferred to [6]. Briefly, it can be shown that the lattice $(\chi, \leq)$ can be chosen as $(\chi, \leq)=$ $(\mathcal{P}(\mathcal{U}), \subseteq)$. Moreover, the function $\tilde{f}: \chi \times \mathcal{Y} \rightarrow \chi$ is defined $\tilde{f}(w, y)=f\left(\alpha_{1}, y\right) \vee \ldots \vee$ $f\left(\alpha_{n}, y\right)$ for any $w=\alpha_{1} \vee \ldots \vee \alpha_{n}$, and $\tilde{f}(\perp, y)=\perp$. Next, lattices $\left(\mathcal{Z}_{E}, \leq\right)$, and $(\mathcal{L}, \leq)$ with extensions $\tilde{h}$ and $\tilde{F}$ that satisfy the desired properties are constructed as well.

Define $\{z \mid y=g(\alpha, z), \alpha \in \mathcal{U}\}:=m(\alpha, y)$. Then $\mathcal{Z}_{E}$ is defined in the following way: (i) $\mathcal{Z} \subseteq \mathcal{Z}_{E}$; (ii) $m(\alpha, y) \in \mathcal{Z}_{E}$ for any $y \in \mathcal{Y}$ and $\alpha \in \mathcal{U}$; (iii) $\mathcal{Z}_{E}$ is invariant under $h$, i.e. if $\bar{z} \in \mathcal{Z}_{E}$, then $h(\alpha, \bar{z}) \in \mathcal{Z}_{E}$ for any $\bar{z} \in \mathcal{Z}_{E}$ and $\alpha \in \mathcal{U}$; (iv) $\mathcal{Z}_{E}$ is closed under finite unions and finite intersections. By construction, $\left(\mathcal{Z}_{E}, \leq\right)$ is a lattice where the order is established by inclusion. Each element in $\mathcal{Z}_{E}$ is either a submanifold of $\mathcal{Z}$ or a union of disjoint submanifolds. Also, $\left(\chi \times \mathcal{Z}_{E}, \leq\right)$ is a lattice with order established component-wise. Define $(\mathcal{L}, \leq):=\left(\mathcal{P}\left(\chi \times \mathcal{Z}_{E}\right), \subseteq\right)$. Obviously, $\chi \times \mathcal{Z}_{E} \subseteq \mathcal{L}$. Any element $q \in \mathcal{L}$ has the form $q=\left(w_{1}, \bar{z}_{1}\right) \vee \ldots \vee\left(w_{k}, \bar{z}_{k}\right)$, where $\bar{z}_{j} \in \mathcal{Z}_{E}$ and $w_{i} \in \chi$.

Define the function $\tilde{F}: \mathcal{L} \times \mathcal{Y} \rightarrow \mathcal{L}$ in the following way. For any $q=\left(w_{1}, \bar{z}_{1}\right) \vee$ $\ldots \vee\left(w_{k}, \bar{z}_{k}\right) \in \mathcal{L}$, define (omit the dependence on $y$ for simplifying notation)

$$
\tilde{F}(q):=\tilde{F}\left(w_{1}, \bar{z}_{1}\right) \vee \ldots \vee\left(\tilde{F}\left(w_{k}, \bar{z}_{n}\right),\right.
$$

where $\tilde{F}\left(w_{i}, \bar{z}_{i}\right):=\left(\tilde{f}\left(w_{i}\right), \tilde{h}\left(w_{i}, \bar{z}_{i}\right)\right)$. Let $w_{i}=\alpha_{i, 1} \vee \ldots \vee \alpha_{i, p_{i}}$ and $\bar{z}_{i}=m_{i, 1} \vee \ldots \vee m_{i, n_{i}}$ with $m_{i, 1}$ submanifolds of $\mathcal{Z}$, then $\tilde{h}: \chi \times \mathcal{Z}_{E} \rightarrow \mathcal{Z}_{E}$ is defined such that $\tilde{h}\left(w_{i}, \bar{z}_{i}\right):=$ $\vee_{l, j} h\left(\alpha_{i, l}, m_{i, j}\right)$. From this definition, it follows that $\tilde{F}$ is order preserving. Also, $\tilde{F}(\perp):=$ $\perp$.

The function $\tilde{g}: \mathcal{L} \rightarrow \mathcal{Y}$ is defined in the following way. For any $q \in \mathcal{L}$ for $q=\left(w_{1}, \bar{z}_{1}\right) \vee \ldots \vee\left(w_{k}, \bar{z}_{k}\right), w_{i}=\alpha_{i, 1} \vee \ldots \vee \alpha_{i, p_{i}}$, and $\bar{z}_{i}=m_{i, 1} \vee \ldots \vee m_{i, n_{i}}$

$$
\tilde{g}(q):=y \text { iff } \tilde{g}\left(w_{i}, \bar{z}_{i}\right)=y,
$$

with $\tilde{g}\left(w_{i}, \bar{z}_{i}\right)=y$ iff $g\left(\alpha_{i, l}, m_{i, j}\right)=y$ for any $l, j$, where $g\left(\alpha_{i, l}, m_{i, j}\right)=y$ if and only if $m_{i, j} \subseteq m\left(\alpha_{i, j}, y\right)$ by definition of $m\left(\alpha_{i, j}, y\right)$.

For any $q=\left(w_{1}, \bar{z}_{1}\right) \vee \ldots \vee\left(w_{k}, \bar{z}_{k}\right) \in \mathcal{L}$, its lower and upper approximations are defined as $a_{L}(q):=\left(w_{1} \wedge \ldots \wedge w_{k}, \bar{z}_{1} \wedge \ldots \wedge \bar{z}_{k}\right)$ and $a_{U}(q):=\left(w_{1} \vee \ldots \vee w_{k}, \bar{z}_{1} \vee \ldots \vee \bar{z}_{k}\right)$. An example of elements in the lattice $(\mathcal{L}, \leq)$ with lower and upper approximations is shown in Figure 2.

The lattices and the system extension have been constructed. Now, the items of Definition 6 and Definition 7 can be checked. Item (i) of Definition 6 is satisfied with $\left[l_{w}, u_{w}\right]=[\perp, w]$ for $w=\alpha_{1} \vee \ldots \vee \alpha_{n}$, with $\alpha_{i}$ such that $g\left(\alpha_{i}, z(k)\right)=y(k)$ and $\tilde{g}\left(f\left(\alpha_{i}, y(k)\right), \tilde{h}\left(\alpha_{i}, z(k)\right)\right)=y(k+1)$. Items (i)-(ii) of Definition 7 are satisfied with $\left\{q \in \mathcal{L} \mid y(k)=\tilde{g}(q), \pi_{1} \circ a_{L}(q)=\perp, \pi_{1} \circ a_{U}(q)=w\right\}=\left[\perp, u_{q}(k, w)\right]$ with $u_{q}(k, w)=$ $\left(\alpha_{1}, m\left(\alpha_{1}, y(k)\right)\right) \vee \ldots \vee\left(\alpha_{n}, m\left(\alpha_{n}, y(k)\right)\right)$ if $w=\alpha_{1} \vee \ldots \vee \alpha_{n}$. Also, $a_{L}(\perp)=\perp$ and $\pi_{2} \circ a_{U}\left(u_{q}(k, w)\right)=m\left(\alpha_{1}, y(k)\right) \vee \ldots \vee m\left(\alpha_{n}, y(k)\right)$.

Item (iii) of Definition 7 is satisfied because $\tilde{F}$ is order preserving by construction and because $\tilde{F}: \alpha \times[\perp, m(\alpha, y)] \rightarrow[\perp, \tilde{F}(\alpha, m(\alpha, y))]$ is one-one because the system is observable. 


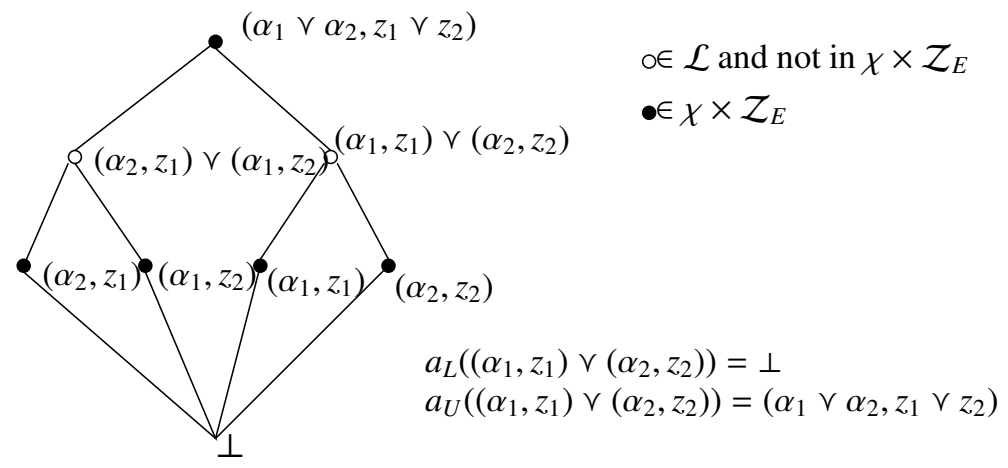

Fig. 2. Hasse diagram representing elements in the lattice $(\mathcal{L}, \leq)$.

To verify (iv) of Definition 7, a distance function on $\mathcal{Z}_{E}$ is defined. For any $\bar{z}_{1}, \bar{z}_{2} \in$ $\mathcal{Z}_{E}$, define

$$
d\left(\bar{z}_{1}, \bar{z}_{2}\right):= \begin{cases}\left|\operatorname{dim}\left(\bar{z}_{1}\right)-\operatorname{dim}\left(\bar{z}_{2}\right)\right| & \text { if } \bar{z}_{1} \text { and } \bar{z}_{2} \text { are related } \\ 1 & \text { if } \bar{z}_{1} \text { and } \bar{z}_{2} \text { are not related, }\end{cases}
$$

where if $\bar{z}=m_{1} \vee \ldots \vee m_{n}, \operatorname{dim}(\bar{z}):=\sum_{i} \operatorname{dim}\left(m_{i}\right)$, and $\operatorname{dim}\left(m_{i}\right)$ denotes the dimension of the submanifold $m_{i} \subset \mathcal{Z}$. Define $\operatorname{dim}(\perp)=0, \operatorname{dim}(z)=1$ for any $z \in \mathcal{Z}$, thus a submanifold isomorphic to $\mathbb{R}^{m}$ has dimension $m+1$. Properties (i)-(ii) of Definition 1 are verified. (Note that any two points in $\mathcal{Z}$ are not related.) To verify (iii) of the Definition 1 , consider $\bar{z}_{1} \leq \bar{z}_{2}$ for $\bar{z}_{1}, \bar{z}_{2} \in \mathcal{Z}_{E}$, and compute $d\left(\perp, \bar{z}_{1}\right)$ and $d\left(\perp, \bar{z}_{2}\right)$. If $\bar{z}_{1} \leq \bar{z}_{2}$, by the way $\mathcal{Z}_{E}$ has been constructed, it means that there are $m_{i}$ and $m_{i}^{\prime}$ submanifolds in $\mathcal{Z}_{E}$ such that $\bar{z}_{1}=m_{1} \vee \ldots \vee m_{n}$, and $\bar{z}_{2}=m_{1}^{\prime} \vee \ldots \vee m_{p}^{\prime}$ with $n \leq p$, and for any $i$ there is a $j$ such that $m_{i} \subseteq m_{j}^{\prime}$. Thus, $\operatorname{dim}\left(\bar{z}_{1}\right)=\operatorname{dim}\left(m_{1}\right)+\ldots+\operatorname{dim}\left(m_{n}\right)$ and $\operatorname{dim}\left(\bar{z}_{2}\right)=\operatorname{dim}\left(m_{1}^{\prime}\right)+\ldots+\operatorname{dim}\left(m_{p}^{\prime}\right)$ with $n \leq p$ and $\operatorname{dim}\left(m_{i}\right) \leq \operatorname{dim}\left(m_{i}^{\prime}\right)$. Thus expression (5) defines a distance function according to Definition 1. Thus, for any $[\perp, U] \subseteq\left[\perp, u_{w}\right]$ with $U=\alpha_{1} \vee \ldots \vee \alpha_{n}$

$$
d\left(\perp, \pi_{2} \circ a_{U} \circ \tilde{F}\left(u_{q}(k, U)\right)\right)=d\left(\perp, h\left(\alpha_{1}, m\left(\alpha_{1}, y\right)\right) \vee \ldots \vee h\left(\alpha_{n}, m\left(\alpha_{n}, y\right)\right)\right),
$$

as $\tilde{F}\left(u_{q}(k, U)\right)=\left(f\left(\alpha_{1}\right), h\left(\alpha_{1}, m\left(\alpha_{1}, y\right)\right) \vee \ldots \vee\left(f\left(\alpha_{n}\right), h\left(\alpha_{n}, m\left(\alpha_{n}, y\right)\right), a_{U} \circ \tilde{F}\left(u_{q}(k, U)\right)=\right.\right.$ $\left(f\left(\alpha_{1}\right) \vee \ldots \vee f\left(\alpha_{n}\right), h\left(\alpha_{1}, m\left(\alpha_{1}, y\right)\right) \vee \ldots \vee h\left(\alpha_{n}, m\left(\alpha_{n}, y\right)\right)\right)$, and thus $\pi_{2} \circ a_{U} \circ \tilde{F}\left(u_{q}(k, U)\right)=$ $h\left(\alpha_{1}, m\left(\alpha_{1}, y\right)\right) \vee \ldots \vee h\left(\alpha_{n}, m\left(\alpha_{n}, y\right)\right)$. Concluding, the definition of distance yields to

$$
d\left(\perp, h\left(\alpha_{1}, m\left(\alpha_{1}, y\right)\right) \vee \ldots \vee h\left(\alpha_{n}, m\left(\alpha_{n}, y\right)\right)\right)=\sum_{i=1}^{n} \operatorname{dim}\left(h\left(\alpha_{i}, m\left(\alpha_{i}, y\right)\right) \leq d_{M}|[\perp, U]|,\right.
$$

where $d_{M}=\max _{i} \operatorname{dim}\left(h\left(\alpha_{i}, m\left(\alpha_{i}, y\right)\right)\right.$.

Remark 1. In the case the system $\Sigma$ is monotone and observable in two steps (see [7]), the same result holds with $\mathcal{Z}_{E}=\mathcal{Z}$ and $\mathcal{L}=\chi \times \mathcal{Z}$. 
This theorem shows that for observable and discrete state observable systems it is always possible to construct the estimator on a lattice proposed in Theorem 1. However, the main advantage of the use of such a method is clear when the space of discrete and/or the space of continuous variables can be extended to lattices where the order relation can be efficiently computed using algebraic properties. This is the case of the monotone deterministic transition systems considered in [7]. To illustrate this point, in the next section three examples are proposed.

\section{Simulation examples}

The first example is a linear hybrid automaton where a lattice of the type constructed in the proof of Theorem 2 is used. The second example is characterized by a continuous dynamics which is monotone (see [10]), and this allows to have $\mathcal{Z}_{E}=\mathcal{Z}$ with a cone partial order. The third example is a multi-robot example proposed in [7], which is a monotone DTS, and thus it allows the largest complexity reduction.

Example 1. Linear discrete time hybrid automaton. Define $\mathcal{U}=\left\{\alpha_{1}, \alpha_{2}, \alpha_{3}, \alpha_{4}, \alpha_{5}\right\}$,
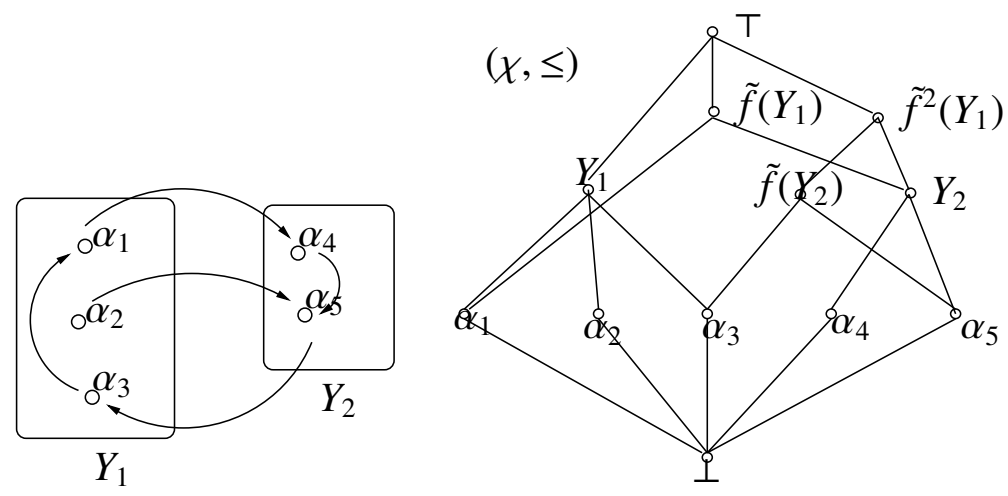

Fig. 3. Map $f$ and output function for the automaton of Example 1 (left). Lattice $(\chi, \leq)$ and the extended function $\tilde{f}$ (right).

and $\alpha(k+1)=f(\alpha(k))$ where $f$ is defined in the Figure 3 left. Assume $\mathcal{Z}=\mathbb{R}^{n}$, $z(k+1)=A(\alpha(k)) z(k)+B(\alpha(k))$, where $A\left(\alpha_{i}\right)=A_{i} \in \mathbb{R}^{n} \times \mathbb{R}^{n}$ and $B\left(\alpha_{i}\right)=B_{i} \in \mathcal{Z}$. The output function $g$ is such that $g(\alpha, z)=\left(g_{\alpha}(\alpha), g_{z}(\alpha, z)\right)$, where $g_{\alpha}: \mathcal{U} \rightarrow\left\{Y_{1}, Y_{2}\right\}$ and $g_{z}(\alpha, z)=C(\alpha) z$, with $C\left(\alpha_{i}\right)=C_{i} \in \mathbb{R}^{m} \times \mathcal{Z}$.

An instance of such an example is considered with $n=3$, where $A_{1}=\left((1,1,1)^{\prime}\right.$, $\left.(0,1,1)^{\prime},(0,0,1)^{\prime}\right)^{\prime}, A_{2}=\left((1 / 2,1 / 2,1 / 2)^{\prime},(1,2,2)^{\prime},(0,0,1)^{\prime}\right)^{\prime}, A_{3}=\left((2,1,1)^{\prime},(0,1,1)^{\prime}\right.$, $\left.(2,0,0)^{\prime}\right)^{\prime}, A_{4}=\left((1,1,1)^{\prime},(1,1,0)^{\prime},(0,0,1)^{\prime}\right)^{\prime}, A_{5}=\left((1,0,0)^{\prime},(1,1,1)^{\prime},(1,1,0)^{\prime}\right)^{\prime}$, $C_{1}=(1,0,0), C_{2}=(1,1,2), C_{3}=(0,0,0), C_{4}=(1,0,0)$, and $C_{5}=(0,1,1)$. The values of $B_{i}$ are not relevant for computing the estimator performance, and thus they are omitted. 
For the discrete state estimate, the minimal lattice $(\chi, \leq)$ where the system is extended is shown in Figure 3 right. Its size is always smaller than $|\mathcal{U}|^{2}$ as pointed out in [6], and its construction is analogous to the construction of the observer tree as done in [4] and [1].

For the continuous state estimate, the lattice $\left(\mathcal{Z}_{E}, \leq\right)$ is constructed according to the proof of Theorem 2, where the submanifolds are affine linear subspaces. Thus, $z_{U}(k)$ at each step $k$ is a collection of affine linear subspaces, each given by the set of $z \in \mathbb{R}^{3}$ such that $M_{i}(k) z=\left(Y(k)-V_{i}(k)\right)$, where $M_{i}(k)=\left(C\left(\alpha_{i}\right)^{\prime},\left(C\left(f\left(\alpha_{i}\right)\right) A\left(\alpha_{i}\right)\right)^{\prime}, \ldots\right.$, $\left.\left(C\left(f^{k-1}\left(\alpha_{i}\right)\right) A\left(f^{k-2}\left(\alpha_{i}\right)\right)\right)^{\prime}\right)^{\prime}, V_{i}(k)=\left(0, C\left(f\left(\alpha_{i}\right)\right) B\left(\alpha_{i}\right), \ldots C\left(f^{k-1}\left(\alpha_{i}\right)\right) B\left(f^{k-2}\left(\alpha_{i}\right)\right)\right)^{\prime}, Y(k)=$ $(y(0), \ldots, y(k-1))^{\prime}$, and $\alpha_{i}$ is such that $f^{k-1}\left(\alpha_{i}\right) \in[\perp, U(k)]$, for $U(k) \in \chi$ and $i \in\{1, \ldots, 5\}$. When only one $\alpha_{i}$ is left in $[\perp, U(k)]$ and the corresponding matrix $M_{i}(k)$ has rank equal to $n$, the estimator has converged. Thus, define $d\left(\perp, z_{U}(k)\right)=\sum_{i=1}^{5} \beta\left(M_{i}(k)\right)$ where

$$
\beta\left(M_{i}(k)\right):= \begin{cases}0 & \text { if } f^{k-1}\left(\alpha_{i}\right) \notin[\perp, U(k)] \\ (n+1)-\operatorname{rank}\left(M_{i}(k)\right) & \text { otherwise }\end{cases}
$$

As a consequence, when $d\left(\perp, z_{U}(k)\right)=1$, the estimator has converged and $z(k)=$ $M_{j}(k)^{\dagger}\left(Y(k)-V_{j}(k)\right)$ for some $j \in\{1, \ldots, 5\}$, where $M_{j}(k)^{\dagger}$ is the pseudoinverse of $M_{j}(k)$. Note that, after the first $k$ at which $d\left(\perp, z_{U}(k)\right)=1$, the state of the system is tracked. The behavior of $d(\perp, U(k)):=|[\perp, U(k)]|$ and of $d\left(\perp, z_{U}(k)\right)$ are illustrated in the left plot of Figure 4. Note that a simultaneous discrete-continuous state estimation allows faster convergence rates of the continuous estimate with respect to the case in which the continuous estimate takes place after the discrete estimate has converged.
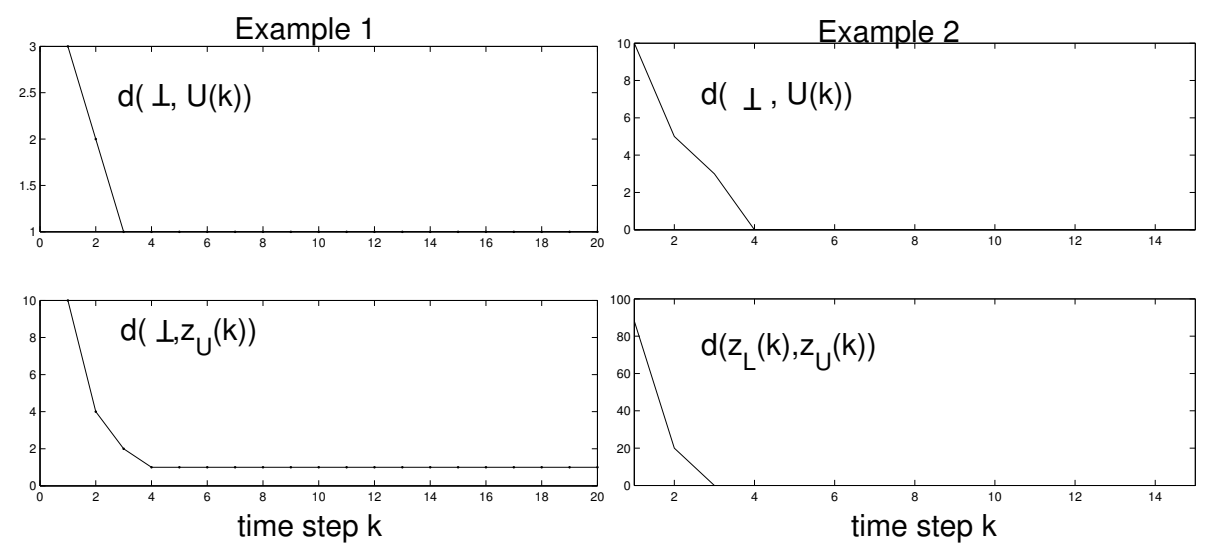

Fig. 4. Estimator performance: example 1 (left) and example 2 (right).

In this example, the continuous variable space does not have monotone properties. As a consequence, the representation of the elements of $(\chi, \leq)$ and of $\left(\mathcal{Z}_{E}, \leq\right)$ involves a listing of objects: for $\chi$, there is a listing of $\alpha_{i}$ s and for $\mathcal{Z}$ we have a listing of linear 
subspaces. Moreover, to represent each linear subspace, a number of constants larger than $n$ (the number of constants needed for representing an element in $\mathbb{R}^{n}$ ) is needed. A measure of the complexity of the estimator is given in the sequel. If $|\mathcal{U}|$ is very large, this choice of the partial orders renders the estimation process prohibitive. A case in which a different partial order must be used for computational tractability, is presented in Example 3.

Example 2. This example considers the case in which it is possible to choose $\mathcal{Z}_{E}=$ $\mathcal{Z}$ because the system is monotone (see [7] for a formal definition). Let again $\mathcal{U}=$ $\left\{\alpha_{1}, \alpha_{2}, \alpha_{3}, \alpha_{4}, \alpha_{5}\right\}$, and $\alpha(k+1)=f(\alpha(k))$ where $f$ is defined in Figure 3 (left). The continuous dynamics is given by

$$
\begin{aligned}
& z_{1}(k+1)=(1-\beta) z_{1}(k)-\beta z_{i, 2}(k)+2 \beta X(\alpha(k)) \\
& z_{2}(k+1)=(1-\lambda) z_{2}(k)+\lambda X(\alpha(k)),
\end{aligned}
$$

where $\beta=0.1, \lambda=0.1, X\left(\alpha_{i}\right):=10 i$ for $i \in\{1, \ldots, 5\}$. The minimal lattice $(\chi, \leq)$ is shown in Figure 3 (right). In this case $\mathcal{L}=\chi \times \mathcal{Z}$, where $\mathcal{Z}=\mathbb{R}^{2}$, and the order $(\mathcal{Z}, \leq)$ is chosen such that $\left(z_{1}^{a}, z_{2}^{a}\right) \leq\left(z_{1}^{b}, z_{2}^{b}\right)$ if and only if $z_{2}^{a} \leq z_{2}^{b}$. The function $\tilde{h}$ : $\chi \times \mathcal{Z} \rightarrow \mathcal{Z}$ is defined by defining the function $\tilde{X}: \chi \rightarrow \mathbb{R}$ in the following way. $\tilde{X}\left(Y_{1}\right):=\max \left(X\left(\alpha_{1}\right), X\left(\alpha_{2}\right), X\left(\alpha_{3}\right)\right)=30, \tilde{X}\left(Y_{2}\right):=\max \left(X\left(\alpha_{3}\right), X\left(\alpha_{5}\right)\right)=50$, and in analogous way for the others, that is $\tilde{X}\left(\tilde{f}\left(Y_{2}\right)\right)=50, \tilde{X}\left(\tilde{f}^{2}\left(Y_{1}\right)\right)=50, \tilde{X}\left(\tilde{f}\left(Y_{1}\right)\right)=50$, and $\tilde{X}(\perp):=0$. With this choice, $\tilde{h}\left(w_{1}, z^{a}\right) \leq \tilde{h}\left(w_{2}, z^{b}\right)$ for any $\left(w_{1}, z^{a}\right) \leq\left(w_{2}, z^{b}\right)$, that is the system is monotone. Convergence plots are shown in Figure 4 (right).

As opposite to Example 1, in this case the representation of the elements in $\mathcal{Z}_{E}$ requires only $n$ scalar numbers, and the computation of the order relation is straightforward. This alleviates the computational burden with respect to the previous example.

Example 3. This example shows the case in which there is a $(\chi, \leq)$, whose order relation can be computed algebraically, and $\mathcal{Z}_{E}=\mathcal{Z}=\mathbb{R}^{20}$, with order established according to the cone order. There are $N=10$ discrete variables updated in a highly coupled fashion (the assignments), each living in the set $\{1, \ldots, N\}$. As a consequence $\mathcal{U}=[1, N]^{N}$. This example is the multi-robot example described in detail in [7], and it is a monotone DTS. Here, only convergence plots are shown, and they are in Figure 5. The size of $\mathcal{U}$ is of the order of $N^{N}$, but thanks to the monotonic properties of the system, the computational complexity of the estimator is linear with $N$.

\section{Complexity considerations}

The scope of the proposed examples is two-fold. First, they give an idea of the range of systems to which the lattice estimation approach applies (just observable and discrete state observable systems). Second, they point out that the lattice approach alleviates the computational burden of the estimator and even renders intractable problems tractable when the system has monotone properties and a good choice of the lattices is made. To make this point more formal, the computational complexity in each of the examples is estimated as function of the continuous variables, the discrete variables, and the sizes of the sets where the discrete variables lie. This section is not meant to be a formal treatment of computational complexity, but has the scope of giving a qualitative measure of 

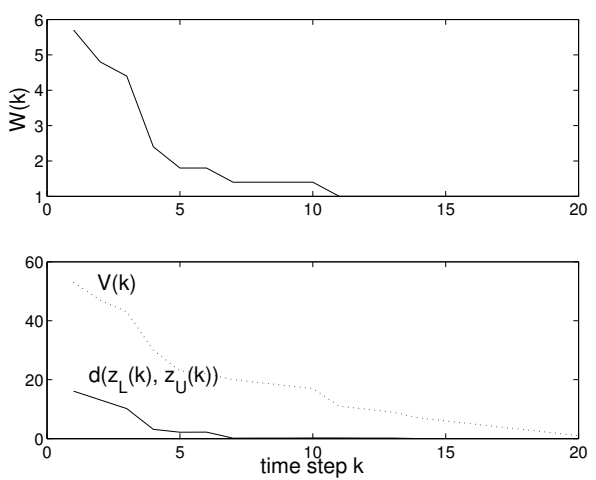

Fig. 5. Estimator performance: example 3. $W(k)$ represents the discrete state estimation error and $V(k)$ is the monotonic function bounding the continuous state estimation error.

the computational complexity diversity of the proposed examples. Let $n$ be the number of continuous variables ( 3 for the first example, 2 for the second, and 20 in the third), $N$ be the number of discrete variables ( 1 in the first example, 1 in the second example, and 10 in the third example), and $u$ be the set where each discrete variable lie (in the first and second example $u=\mathcal{U}$, and in the third $u=\{1, \ldots, N\}$ and $\left.\mathcal{U}=u^{N}\right)$. The computational cost of the estimator is computed as

$$
\text { computational cost } \propto S+a_{U C}
$$

where $S$ is the sum of the sizes of the look-up tables used at each update of the estimator, and $a_{U C}$ is the algebraic update cost of each estimator update. The cost of any set of algebraic computation is set to 1 . One can verify that $S \propto|u|^{2 N}$ in the first two examples, and that $S \propto 2 N$ in the third one. In the first example, $a_{U C} \propto|u|^{N} n$, and $a_{U C} \propto 2 n$ in the second and third examples. This is shown in the following table.

Table 1: Estimator computational cost

$$
\begin{array}{l|l}
\hline \text { Example 1 } & |u|^{2 N}+|u|^{N} n \\
\hline \text { Example 2 } & |u|^{2 N}+2 n \\
\hline \text { Example 3 } & 2 N+2 n \\
\hline &
\end{array}
$$

From the table, one notice that moving from Example 1 to Example 3 the computational burden due to the size of $u$ decreases, and it disappears in the case of the third example. This is due to the monotone properties of the continuous dynamics in Example 2 and Example 3, and to the existence of a lattice $(\chi, \leq)$ with algebraic properties in Example 3 . Note also that the complexity reduction that characterizes the third example does not occur because the discrete variables dynamics decouples, as in fact it is heavily coupled. 


\section{Conclusions}

In this paper, a cascade discrete-continuous state estimator design is proposed under observability and discrete state observability assumptions. As pointed out also in the simulation examples section, the proposed approach is general. The main advantage of using a lattice approach to the estimation problem is clear when the system has monotone properties that can be exploited in the estimator construction. In this case, the computational complexity is drastically reduced and a scalability property holds in the number of variables to be estimated. Thanks to this feature, the estimator can be efficiently designed even for systems with large discrete state spaces, for which the state estimation problem is intractable if the monotone properties are not directly exploited (see Example 3).

The results obtained in this paper suggest that a partial order structure is a possible way for overcoming complexity issues in the estimation of hybrid systems. A future research trust will try to generalize these ideas to the coupled discrete-continuous state estimation problem. Given the promising results obtained using partial order theory for state estimation problems, the authors will explore the possibility of applying similar tools for other control and analysis problems in hybrid systems.

\section{References}

1. A. Balluchi, L. Benvenuti, M. D. Di Benedetto, and A. Sangiovanni-Vincentelli. Design of observers for hybrid systems. In Lecture Notes in Computer Science 2289,C. J. Tomlin and M. R. Greensreet Eds. Springer Verlag, pages 76-89, 2002.

2. Alexandre Bayen, Jiawei Zhang, Claire Tomlin, and Yinyu Ye. Milp formulation and polynomial time algorithm for an aircraft scheduling problem. In Proc. of American Control Conference, 2003.

3. A. Bemporad, G. Ferrari-Trecate, and M. Morari. Observability and controllability of piecewise affine and hybrid systems. IEEE Transactions on Automatic Control, 45:1864-1876, 1999.

4. P. E. Caines. Classical and logic-based dynamic observers for finite automata. IMA J. of Mathematical Control and Information, pages 45-80, 1991.

5. D. DelVecchio and R. M. Murray. Discrete state estimators for a class of hybrid systems on a lattice. In Lecture Notes in Computer Science 2993, R. Alur and G. Pappas Eds. Springer Verlag, pages 311-325, 2004.

6. D. DelVecchio and R. M. Murray. Existence of discrete state estimators for hybrid systems on a lattice. In Conf. on Decision and Control, 2004.

7. D. DelVecchio and R. M. Murray. Cascade discrete-continuous state estimators for a class of monotone systems. In IFAC (Submitted), pages 76-89, 2005.

8. J. E. Hopcroft, R. Motwani, and J. D. Ullman. Introduction to Automata Theory, languages, and Computation. Addison Wesley, 2001.

9. A. Jung S. Abramsky. Domain Theory. Handbook of Logic in Computer Science Volume 3. 1994.

10. H. L. Smith. Monotone Dynamical Systems. Mathematical Surveys and Monographs. American Mathematical Society, 1995.

11. Claire Tomlin, Ian Mitchell, , and Ronojoy Ghosh. Safety verification of conflict resolution maneuvers. IEEE Transactions on Intelligent Transportation Systems, 2001.

12. R. Vidal, A. Chiuso, and S. Soatto. Observability and identifiability of jump linear systems. In Conf. on Decision and Control, pages 3614 - 3619, Las Vegas, 2002. 